Journal of Animal and Veterinary Advances 11 (10): 1727-1733, 2012

ISSN: $1680-5593$

(C) Medwell Journals, 2012

\title{
Identification, Cloning, Expression of a Novel Functional Anas platyrhynchos mRNA TLR4
}

\author{
${ }^{1,2}$ Wael K. Elfeil, ${ }^{3,4}$ Reham R. Abouelmaatti, ${ }^{1}$ Changjiang Sun, ${ }^{1}$ Wenyu Han, ${ }^{3}$ Xiaokun Li, \\ ${ }^{3}$ Jisheng Ma, ${ }^{1}$ Liancheng Lei, ${ }^{1}$ Shanshan Liu, ${ }^{1}$ Yongjun Yang, ${ }^{1}$ Yu Wang, \\ ${ }^{2}$ Mostafa Mandour, ${ }^{2}$ Mohamed Fawzy and ${ }^{3,5}$ Mohamed N. Shalaby \\ ${ }^{1}$ College of Veterinary Medicine and Animal Science, Jilin University, Changchun, 130062 Jilin, China \\ ${ }^{2}$ Faculty of Veterinary Medicine, Suez Canal University, 41522 Ismailia, Egypt \\ ${ }^{3}$ Norman Bethune College of Medicine, Jilin University, Changchun, 130021 Jilin, China \\ ${ }^{4}$ Key Laboratory of Animal Epidemiology and Zoonosis, Sharqia Vet. Directorate, \\ General Org. Vet. Services, Ministry of Agriculture, Egypt \\ ${ }^{5}$ College of Physical Education, Portsaid University, Egypt
}

\begin{abstract}
Toll like receptor mediate immune response through recognition of Pathogen-Associated Molecular Patterns (PAMPs) thus play important roles in host defense. Little is known about the Avian Immune System structure and the majority of it based on chicken as a Universal Avian Model while recent researches showed that a lot of factor not common between chicken and other birds. This research aimed to identify and clone the duck TLR4 as a model to Anseriformes species, researchers success to clone a novel functional dTLR4 mRNA and deposit into the NCBI GenBank database where the mRNA consist of 2529 nucleotide and it showed 81\% identity with chicken and $78 \%$ identity with zebra finch while showed $95 \%$ identity with goose. The translated amino acid showed that the gene consist of 843 amino acid and the transmembrane structure analysis reveal a novel unique LRR motifs in duck and absent in both chicken and zebra finch at the position 56-80 also showed that TIR domain consist of 146 amino acid not 145 like chicken and zebra finch.
\end{abstract}

Key words: Avian, Anseriformes, duck-toll like receptor, TLR4, gene expression, China

\section{INTRODUCTION}

Pathogen-Associated Molecular Patterns or PAMPs are molecules associated with groups of pathogens that are recognized by cells of the Innate Immune System. These molecules can be referred to as small molecular motifs conserved within a class of microbes. They are recognized by Toll-Like Receptors (TLRs) and other Pattern Recognition Receptors (PRRs) in both plants and animals. They activate innate immune responses, protecting the host from infection by identifying some conserved non-self-molecules. Bacterial Lipopolysaccharide (LPS), an endotoxin found on the bacterial cell membrane of a bacterium is considered to be the prototypical PAMP. Every TLR receptor mainly specialized in recognizing one or more pattern where LPS is specifically recognized by TLR4 (Faure et al., 2001; Kaiser, 2007; Kogut et al., 2005; Leveque et al., 2003; Pan et al., 2012; Vinkler et al., 2009; Zhang et al., 2011), a bacterial flagellin recognized by TLR5 (Kogut et al., 2005), lipoteichoic acid from gram positive bacteria and peptidoglycan recognized by TLR2 (Boyd et al., 2001; Faure et al., 2001; Fukui et al., 2001) and nucleic acid variants normally associated with viruses such as double-stranded RNA dsRNA recognized by TLR3 (Brownlie and Allan, 2011; Miggin and O'Neill, 2006; Roach et al., 2005) or unmethylated $\mathrm{CpG}$ motifs, recognized by TLR15, 21 (Alcaide and Edwards, 2011; He et al., 2012; Li et al., 2012; Ramasamy et al., 2011; Werling et al., 2009) Although, the term PAMP is relatively new, the concept that molecules derived from microbes must be detected by receptors from multicellular organisms has been held for many decades and references to an endotoxin receptor are found in much of the older literature. Toll-Like Receptors (TLRs) are the basic components of the vertebrate pathogen recognition system. Despite uniform general structure, remarkable variability in domain composition can be found in individual TLRs among species. Knowledge of interspecific differences is of particular importance to the understanding of selective pressures on TLRs. Toll-Like Receptors (TLRs) are membrane-bound sensors of the

Corresponding Author: Han Wenyu, Lab of Microbiology and Immunology, College of Veterinary Medicine and Life Science, Heping Campus, Jilin University, 5333 Xi' an Road, 130062 Changchun, China 
Innate Immune System that recognize invariant and distinctive molecular features of invading microbes and are also essential for initiating adaptive immunity in vertebrates. The genetic variation at $T L R$ genes has been directly related to differential pathogen outcomes in humans and livestock. Nonetheless, new insights about the impact of TLRs polymorphism on the evolutionary ecology of infectious diseases can be gained through the investigation of additional vertebrate groups not yet investigated in detail. Toll-Like Receptors (TLRs) are a member of the Pattern Recognition Receptors (PRRs) which detect Pathogen-Associated Molecular Patterns (PAMPs) and have a role in initiating the innate as well as adaptive immune defense (Alcaide and Edwards, 2011; Brownlie and Allan, 2011; Chausse et al., 2011; Faure et al., 2001; He et al., 2012; Jin and Lee, 2008; Miggin and O'Neill, 2006; De La Lastra and De La Fuente, 2007; Roach et al., 2005; Ruan et al., 2012; Temperley et al., 2008; Yilmaz et al., 2005). They play a vital role in host immune responses through the recognition of Lipopolysaccharides (LPS), lipopeptides, flagellins, dsRNA or CpG DNA motifs (Werling et al., 2009). TLR System is part of ancient machinery that is evolutionary conserved with homologs present in insects, nematodes, plants, fish, mammals and birds (Beutler and Rehli, 2002). A range of $T L R$ genes has been identified in non-mammalian vertebrates including chicken and fish (Roach et al., 2005). So far, at least 10 members of the avian TLRs family have been identified including TLR1 Type 1 and 2, TLR2 Type 1 and 2, TLR3, 4, 5, 7, 15 and 21 . The duplicated genes, TLR1 Type 1 and 2 that were found in avian are the orthologs of TLR1 and TLR6 of mammals and TLR2 Type 1 and 2 in avian are orthologs to the single TLR2 of mammals. Avian TLRs 3, 4, 5 and 7 are directly orthologs to those in other vertebrates. TLR15 is unique to avian and avian TLR21 is an ortholog of TLR21 in fish and amphibians (Acevedo-Whitehouse and Cunningham, 2006; Alcaide and Edwards, 2011; Brownlie and Allan, 2011). Currently, most TLRs are characterized only in a limited number of model species including domestic chicken as a Universal Avian Model. Here, researchers want to describe the TLRs in duck. The research dedicated to the description of the enormous diversity of molecules involved in pathogen recognition is of vital importance in human and veterinary medicine. It is equally important to the evolutionary biology of host-parasite interactions. Much effort has been devoted to the characterization of Immune System components in human and mouse models while much less is currently known about the architecture of the Immune System in other species (Acevedo-Whitehouse and Cunningham,
2006). Information concerning any one of the wealth of living species may bring new insights into the principles of vertebrate immune function. Aiming to describe general patterns of Immune System evolution in terrestrial vertebrates, the investigation of the bird clade may be particularly useful. Birds form a well-diversified taxon with origin distinct to mammals but with physiology comparable to them. However, in contrast to mammals, the knowledge of the molecular structure of the Avian Immune System is limited (Kaiser, 2007). At present, a single avian species model is available, the domestic chicken (Gallus gallus domesticus). The research on avian major histocompatibility complex has shown that chicken may possess immunogenetic traits that are not common to birds in general (Kelley et al., 2005). Moreover, Galliformes are the representatives of one of the oldest evolutionary branches of modern birds distinct from other avian taxa (Livezey and Zusi, 2007). Considering these reasons, new models are required to verify the universal validity of the results obtained in chicken, so researchers choice duck as a represented to the Anseriformes where it show much difference in the immune response to a lot of disease from chicken or other Galliformes.

\section{MATERIALS AND METHODS}

Samples: Spleen, lung, cecal tonsil, liver, duodenum, muscle, cecum and skin samples were collected from mallard duck. Mallard duck brought as a live mature duck from common farm and kept under inspection for 5 days to be sure it free from any clinical infection, then the sample collected and stored in $-80^{\circ} \mathrm{C}$.

Primer design: Researchers downloaded the complete mRNA sequences of the chicken TLR4 from the GenBank (TLR 4NM_001030693) and zebra finch (TLR4EU779825). The sequence was aligned using the ClustalW application (PrimerPremier5.1 Software) where researchers designed degenerative primers match with the alignment of the sequences to clone short sequence then we got the full sequence by RACE System SMARTer RACE cDNA Amplification kit (Clontech, CA, USA) following the instruction of manufacturer, to get the full length for both direction $3^{\prime}$ end and $5^{\prime}$ end direction all the primers were shown in the Table 1.

Molecular cloning of duck TLR4: Total RNA from duck spleen, muscle, lung, skin, liver, cecal tonsil, duodenum and cecum were extracted by TRIZOL (Invitrogen, USA) according to the manufacture's protocol. Both the quantity and quality of total RNA were assessed at OD260 and 280 using a NanoDrop 
Table 1: List of primers used in all the PCR reactions

\begin{tabular}{ll}
\hline Primer names & Primer sequence \\
\hline 18 S housekeeping gene $F$ & TTGGTGGAGCGATTTGTC \\
18S housekeeping gene $R$ & ATCTCGGGTGGCTCAACG \\
Degenerative dTLR4 primer F 1 & GCCACAACAGCATCACTTC \\
Degenerative dTLR4 primer F 2 & GGAGATCACCCCTAACACC \\
Degenerative dTLR4 primer F 3 & AAACACTGGTCTCAGAAGC \\
Degenerative dTLR4 primer R 1 & CAGCTCCTGCTTCTCCTTG \\
Degenerative dTLR4 primer R 2 & ATCAGGGCATCAAGGTCTC \\
Degenerative dTLR4 primer R 3 & GAGCTGGAGCAGAACCTC \\
RACE dTLR4 primer 3' end direction & GCCCTGGAAATCCTGCCTGACAGTCTGG \\
RACE dTLR4 primer 5' end direction & ACTGTGGTTGGTGGGATGGCAAGAGG \\
Full length dTLR4 primer F & TGCTGAAATCCCAAACACCACC \\
Full length dTLR4 primer R & AGCTTTGTCTCACGGGTCAC \\
\hline
\end{tabular}

ND-1000 spectrophotometer (NanoDrop Technologies, Wilmington, USA). RNA samples were used for synthesis cDNA library in $10 \mu \mathrm{L}$ reaction mixture using BioRT cDNA first Strand Synthesis kit (Hang zhou Bioer, China) according to the manufacture instruction, briefly, oligo dT primer $(0.5 \mu \mathrm{g})$ was used to reverse transcribe $1 \mu \mathrm{g}$ of respective RNA in the presence of dNTP's $(250 \mu \mathrm{M})$, reverse transcriptase buffer (50 $\mathrm{mM}$ Tris- $\mathrm{HCl}$, $100 \mathrm{mM} \mathrm{KCl}, 4.0 \mathrm{mM}$ DTT and $10 \mathrm{mM} \mathrm{MgCl}_{2}$ ), AMV Reverse Transcriptase (5 units $\mu \mathrm{g}^{-1}$ ) and RNasin Ribonuclease inhibitor ( 40 unit $\mu \mathrm{L}^{-1}$ ) at $42^{\circ} \mathrm{C}$ for $45 \mathrm{~min}$ following inactivation at $95^{\circ} \mathrm{C}$ for $5 \mathrm{~min}$. The PCR was performed to amplify the target gene using specific primers where $25 \mu \mathrm{L}$ PCR mixtures contained $50 \mathrm{pmol}$ for each forward and reverse primers, $2 \mu \mathrm{L}$ template cDNA, $200 \mu \mathrm{M}$ each of dNTP mix and $2.5 \mathrm{U}$ Ex Taq polymerase (Takara bio Dalian, china) in $1 \times$ Ex buffer. Amplification conditions were as follows: initial denaturation at $94^{\circ} \mathrm{C}$ for $5 \mathrm{~min}, 35$ cycles at $94^{\circ} \mathrm{C}$ for $35 \mathrm{sec}$, annealing at $56-60^{\circ} \mathrm{C}$ for $35 \mathrm{sec}$ and extension at $72^{\circ} \mathrm{C}$ for $2 \mathrm{~min}$ followed by a final extension at $72^{\circ} \mathrm{C}$ for $10 \mathrm{~min}$. PCR amplicons were verified by $1.2 \%$ agarose gel electrophoresis in TBE buffer at $70 \mathrm{~mA}$ for $45 \mathrm{~min}$ and products visualized by staining with ethidium bromide, check the band and get the images with GelDoc ${ }^{\mathrm{TM}} \mathrm{XR}+$ system (Bio-RAD, USA). Researchers used SMARTer RACE cDNA Amplification kits (Clontech, USA) according to the manufacture instruction to get the full length toward the $3^{\prime}$ end and 5 ' end.

Sequence analysis: The sequence of the duck TLR4 mRNA was blast in the GenBank using nucleotide blast and the translated amino acids also blast by protein blast to check either the new sequence is related to any other cloned gene. The sequence of duck TLR4 was compared with the know TLR4 mRNA sequences from different species which downloaded from the GenBank and aligned by CUSTALW, MEGA 5 Software (Tamura et al., 2011). A phylogenetic tree was constructed from the amino acid alignments using two method:
- The Neighbour-Joining Method with options of pairwise deletion, poisson correction and different evolutionary rates with a gamma parameter of 1

- The Maximum Parsimony Method using the close Neighbour-Interchange Search Method with random additive trees (10 replicates), all the phylogenetic analysis were made by MEGA5 Software and the reliability of branching was tested by 1000 bootstraps (Tamura et al., 2011). The extracellular, transmembrane and cytoplasmic domains of these protein sequences were predicted with the analysis tools provided at the web site (http://smart.embl-heidelberg.de and http://split. pmfst.hr)

\section{RESULTS AND DISCUSSION}

Duck dTLR4: The complete mRNA sequence of duck TLR4 was deposit in the NCBI GenBank database under Accession No.: JN618073. Where it consist of 2529 nucleotide and the consensus cDNA sequence showed $81 \%$ identity with the chicken TLR 4 mRNA sequence and $78 \%$ identity with zebra finch TLR $4 \mathrm{mRNA}$ sequence while showing $39.6 \%$ identity with chicken TLR2-A and lower percent with other chicken TLRs which confirmed the new sequence is probably homologue to chicken TLR4. The predicted protein of encoded by dTLR 4 mRNA sequence is composed of 843 amino acids where it beginning with (ATG) which similar to chicken TLR4 sequence. The dTLR4 domain structure have been estimated using SMART web tool where dTLR4 is formed by large ectodomain, transmembrane domain (22 amino acid) and cytoplasmic Toll/Interlukin-1 Receptor (TIR) domain (146 amino acid). A single peptide (26 amino acid) was shown at the N-terminal part of the ectodomain, it followed by nine LRRs (eight LRRs clear and one detected by Blast and one LRR is unique to duck sequence only at the position 56-80 while it absent in both chicken and zebra finch receptor) that are flanked by Leucine-Rich Repeat Carboxy-Terminal domain (LRRCT) from the other side (50 amino acid) (Fig. 1). 


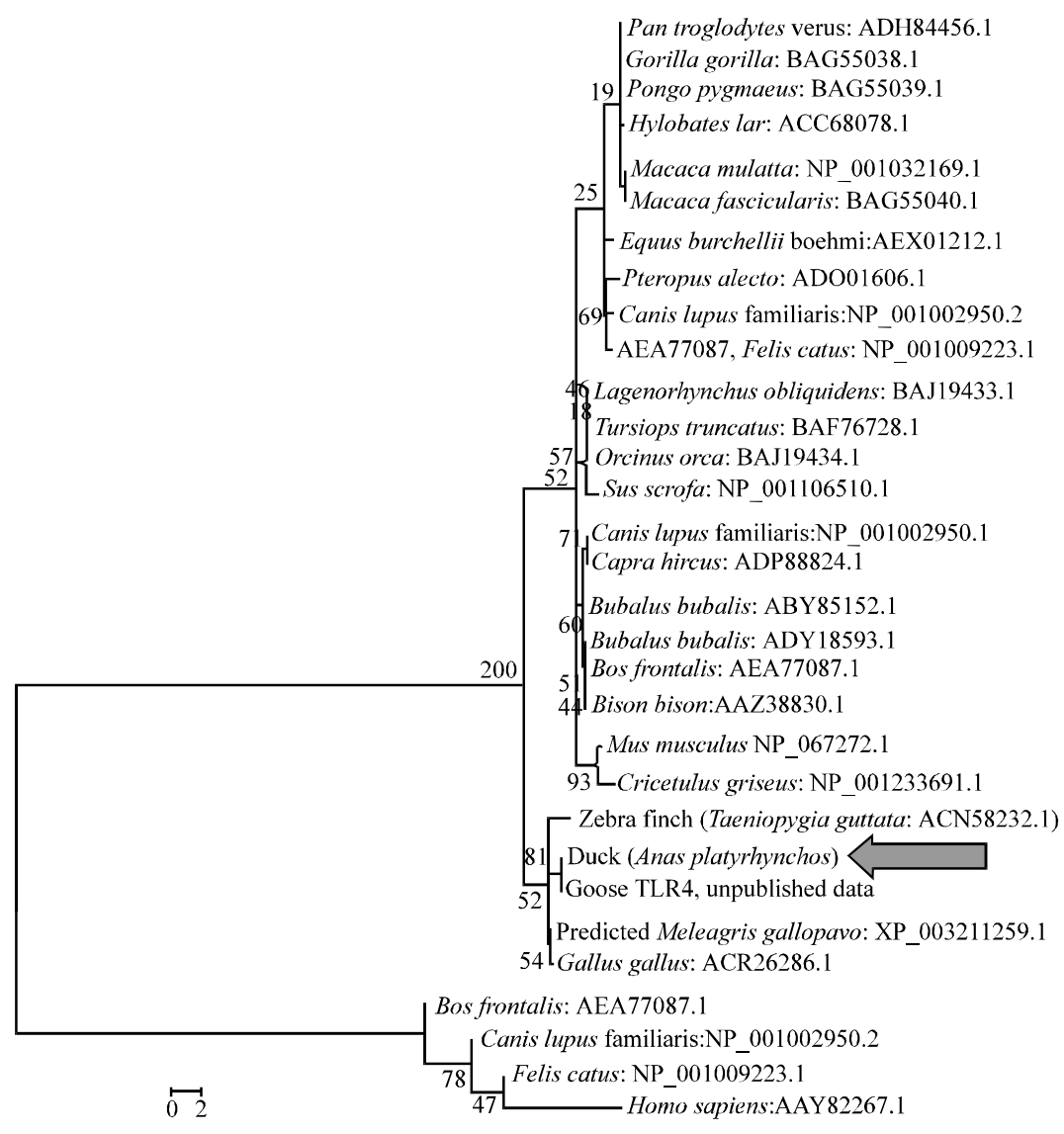

Fig. 1: Phylogenetic tree reconstruction of the dTLR4 repertoire based on the Neighbor-Joining Method. This phylogeny shows the duck TLR4 sequences fron this study plus goose (unpublished data against all know vertebrate TL4. Bootstraps support values for the main branches defining different genes are shown) the duck highlighted by bold arrow infront of it

Similarity with other TLRs: Researchers used two method to construct a phylogenetic tress (NeighbourJoining and Maximum Parsimony) based on amino acid of TLR4 which downloaded from the GenBank. The phylogenetic analysis had been performed using the translated duck and goose amino acid sequence with almost all the know amino acid sequence founded in the GenBank which include the following species: Mus musculus NP_067272, Canis lupus familiaris: NP 001002950, Equus burchellii boehmi: AEX01212, Macaca mulatta: NP_001032169, Ovis aries: NP_001129402, Sus scrofa: NP_001106510, Cricetulus griseus: NP_001233691, Bos frontalis: AEA77087, Felis catus. NP 001009223, Meleagris gallopavo: XP_003211259, Bubalus bubalis: ADY18593, Orcinus orca: BAJ19434, Lagenorhynchus obliquidens: BAJ19433, Capra hircus: ADP88824, Pteropus alecto: AD001606, Gallus gallus: ACR26286, Pan troglodytes verus: ADH84456, Homo sapiens:AA Y82267, Bos taurus: AAQ62700, Taeniopygia guttata: ACN58232, Macaca fascicularis: BAG55040, Pongo pygmaeus: BAG55039, Gorilla gorilla: BAG55038, Pan paniscus: BAG55037, Cercocebus atys: ABY64991, Hylobates lar: ACC68078, Bubalus bubalis: ABY85152, Tursiops truncatus: BAF76728, Bison bison: AAZ38830, both phylogenetic method provided almost results where the phylogenetic analysis showed that dTLR4 is closely related to gTLR4 (goose TLR4), chicken TLR4 and zebrafinch TLR4, respectively. The structure of dTLR4 amino acids as a general similar to other identified TLR4 sequence with $36-48 \%$ identity to different mammalian, $78 \%$ identity to zebrafinch, $81 \%$ identity to chicken and $94 \%$ identity to goose one.

The protein length of dTLR 4 is similar to the chicken one, 843 amino acid, sharing the same starting and ending amino acid. The SMART analysis identified a unique LRR 
Chicken transmembrance structure
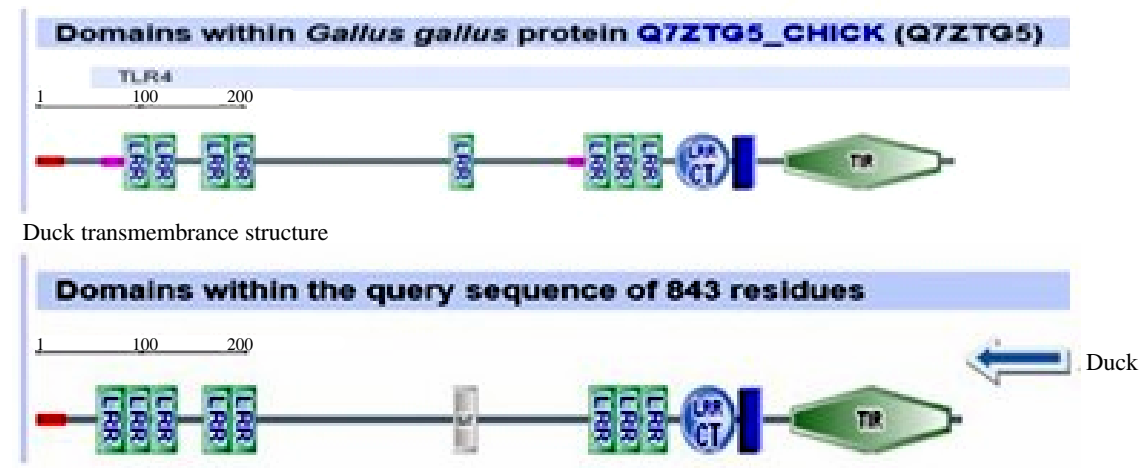

Zebra transmembrance structure

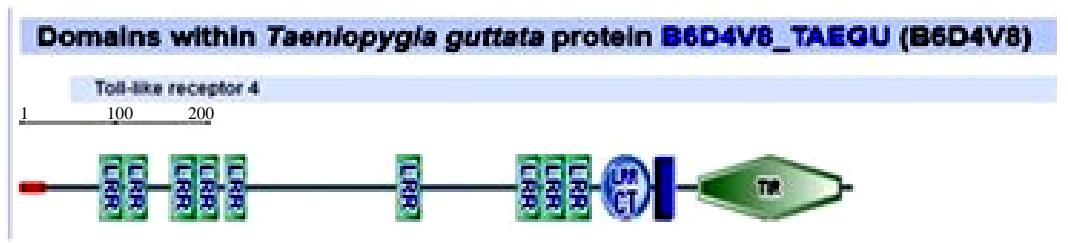

Fig. 2: Transmembrance structure analysis by SMART web tool for chicken, duck and zebra finch TLR4 and duck highlighted by left arrow, where the duck TIR domain has a1 46 amino acid while chicken and zebra finch has a 145 amino acids and it may be due to the unequal force distribution during the evolution also the duck showed a unique LRR domain at the position 56-80 which absent in chicken and zebra finch

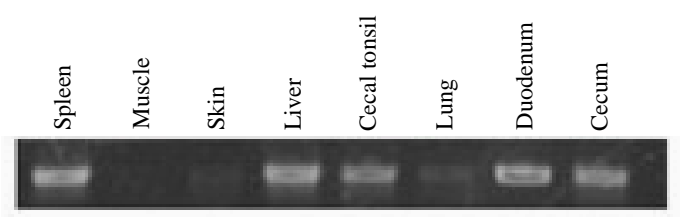

Fig. 3: Tissue expression of dTLR4 mRNA (tissues obtained mature duck and subjected to RT-PCR using primers as shown in Table 1 . The data are representative of three replicate experiments)

motive in duck at the position 56-80 which absent in both chicken and zebra finch while zebra finch has LRR at the position 207-229 which absent in duck and chicken (Fig. 2), these differences maybe a result of unequal selection during the avian evolution and it maybe have a role in the varying in the pathogen response between the avian.

Expression pattern of dTLR4: The transcription of dTLR4 was highly expressed in spleen, cecal tonsil, liver cecum and moderate in lung and duodenum while absent in muscle and skin (Fig. 3), semiquantitves PCR following the reverse transcription showed differences in the expression level among the tested tissue where the expression was highest in spleen, cecal tonsil and liver also showed moderate expression level in lung and intestine.

\section{CONCLUSION}

This is the first study to characterize the duck TLR4 (dTLR4) as most studies focus on non-avian vertebrates and in avian focus on chicken as a General Avian Model, the result provided a dTLR4 which consider a homologue for chicken and other mammalian TLR4, the structure analysis showed that the duck receptor very closely to goose one with $95 \%$ identity and more closer to chicken than to zebra finch.

The transmembrane structure analysis for the duck receptor showed that it has the same amino acid number like chicken and one amino acid plus zebra finch and with chicken and zebra finch sharing the same signal peptide at the begin of the gene. The duck TIR domain has a 146 amino acid while chicken and zebra finch has a 145 amino acids and it may be due to the unequal force distribution during the evolution also the duck showed a unique LRR domain at the position 56-80 which absent in chicken and zebra finch. Which may be has role in the difference of the immune response against pathogen among the duck and other Galliformes species. The expression of dTLR4 varies between chicken and zebra finch where it expressed significantly in cecal tonsil and intestine as in chicken (Leveque et al., 2003; Yilmaz et al., 2005) and in spleen as in zebrafinch (Vinkler et al., 2009) and absent in skin and muscle as in chicken (Yilmaz et al., 2005). 


\section{REFERENCES}

Acevedo-Whitehouse, K. and A.A. Cunningham, 2006. Is $\mathrm{MHC}$ enough for understanding wildlife immunogenetics? Trends. Ecol. Evol., 21: 433-438.

Alcaide, M. and S.V. Edwards, 2011. Molecular evolution of the toll-like receptor multigene family in birds. Mol. Biol. Evol., 28: 1703-1715.

Beutler, B. and M. Rehli, 2002. Evolution of the TIR, tolls and TLRs: Functional inferences from computational biology. Curr. Top. Microbiol. Immunol., 270: 1-21.

Boyd, Y., M. Goodchild, S. Morroll and N. Bumstead, 2001. Mapping of the chicken and mouse genes for Toll-Like Receptor 2 (TLR2) to an evolutionarily conserved chromosomal segment. Immunogenetics, 52: 294-298.

Brownlie, R. and B. Allan, 2011. Avian toll-like receptors. Cell Tissue Res., 343: 121-130.

Chausse, A.M., O. Grepinet, E. Bottreau, Y. Le Vern and P. Menanteau et al., 2011. Expression of Toll-like receptor 4 and downstream effectors in selected cecal cell subpopulations of chicks resistant or susceptible to Salmonella Carrier State. Infection Immun., 79: 3445-3454.

De La Lastra, J.M. and J. De La Fuente, 2007. Molecular cloning and characterisation of the griffon vulture (Gyps fulvus) toll-like receptor 1. Dev. Comp. Immunol., 31: 511-519.

Faure, E., L. Thomas, H. Xu, A. Medvedev, O. Equils and M. Arditi, 2001. Bacterial lipopolysaccharide and IFN- $\gamma$ induce Toll-like receptor 2 and Tolllike receptor 4 expression in human endothelial cells: Role of NF- $\mathrm{BB}$ activation. J. Immunol., 166: 2018-2024.

Fukui, A., N. Inoue, M. Matsumoto, M. Nomura and $\mathrm{K}$. Yamada et al., 2001. Molecular cloning and functional characterization of chicken toll-like receptors. A single chicken toll covers multiple molecular patterns. J. Biol. Chem., 276: 47143-47149.

He, H., K.J. Genovese, C.L. Swaggerty, K.M. Mackinnon and M.H. Kogut, 2012. Co-stimulation with TLR3 and TLR21 ligands synergistically up-regulates Th1cytokine IFN-gamma and regulatory cytokine $\mathbb{L}-10$ expression in chicken monocytes. Dev. Comp. Immunol., 36: 756-760.

Jin, M.S. and J.O. Lee, 2008. Structures of the toll-like receptor family and its ligand complexes. Immunity, 29: $182-191$.

Kaiser, P., 2007. The avian immune genome-a glass halffull or half-empty? Cytogenet Genome Res., 117: $221-230$.
Kelley, J., L. Walter and J. Trowsdale, 2005. Comparative genomics of major histocompatibility complexes. Immunogenetics, 56: 683-695.

Kogut, M.H., M. Iqbal, H. He, V. Philbin, P. Kaiser and A. Smith, 2005. Expression and function of tolllike receptors in chicken heterophils. Dev. Comp. Immunol., 29: 791-807.

Leveque, G., V. Forgetta, S. Morroll, A.L. Smith and N. Bumstead et al., 2003. Allelic variation in TLR4 is linked to susceptibility to Salmonella enterica serovar Typhimurium infection in chickens. Infect. Immunity, 71: 1116-1124.

Li, Y.W., X.C. Luo, X.M. Dan, W. Qiao, X.Z. Huang and A.X. Li, 2012. Molecular cloning of orange-spotted grouper (Epinephelus coioides) TLR21 and expression analysis post Cryptocaryon irritans infection. Fish Shellfish Immunol., 32: 476-781.

Livezey, B.C. and R.L. Zusi, 2007. Higher-order phylogeny of modern birds (Theropoda, Aves: Neornithes) based on comparative anatomy. II. Analysis and discussion. Zool. J. Linnean Soc., 149: 1-95.

Miggin, S.M. and L.A. ONeill, 2006. New insights into the regulation of TLR signaling. J. Leukoc. Biol., 80: $220-226$.

Pan, Z., Q. Fang, S. Geng, X. Kang, Q. Cong and X. Jiao, 2012. Analysis of immune-related gene expression in chicken peripheral blood mononuclear cells following Salmonella enteric serovar Enteritidis infection In vitro. Res. Vet. Sci., 10.1016/j.rvsc. 2011. 12.018

Ramasamy, K.T., P. Verma, M.R. Reddy and S. Murugesan, 2011. Molecular characterization of coding sequence and mrna expression pattern of tolllike receptor 15 in japanese quail (Coturnix Japonica) and indigenous chicken breeds (Aseel and Kadaknath). J. Poul. Sci., 48: 168-175.

Roach, J.C., G., Glusman, L. Rowen, A. Kaur and M.K. Purcell et al., 2005. The evolution of vertebrate Toll-like receptors. Proc. National Acad. Sci., 102: 9577-9582.

Ruan, W.K., Y.H. Wu, J. An, D.F. Cui, H.R. Li and S.J. Zheng, 2012. Toll-like receptor 2 type 1 and type 2 polymorphisms in different chicken breeds. Poult. Sci., 91: 101-106.

Tamura, K., D. Peterson, N. Peterson, G. Stecher, M. Nei and S. Kumar, 2011. MEGA5: Molecular evolutionary genetics analysis using maximum likelihood, evolutionary distance and maximum parsimony methods. Mol. Biol. Evol., 28: 2731-2739.

Temperley, N.D., S. Berlin, I.R. Paton, D.K. Griffin and D.W. Burt, 2008. Evolution of the chicken Toll-like receptor gene family: A story of gene gain and gene loss. BMC Genomics, Vol. 9. 10.1186/1471-2164-9-62. 
Vinkler, M., A. Bryjova, T. Albrecht and J. Bryja, 2009. Identification of the first Toll-like receptor gene in passerine birds: TLR4 orthologue in zebra finch (Taeniopygia guttata). Tissue Antigens, 74: 32-41.

Werling, D., O.C. Jann, V. Offord, E.J. Glass and T.J. Coffey, 2009. Variation matters: TLR structure and species-specific pathogen recognition. Trends Immunol., 30: 124-130.
Yilmaz, A., S. Shen, D.L. Adelson, S. Xavier and J.J. Zhu, 2005. Identification and sequence analysis of chicken Toll-like receptors. Immunogenetics, 56: 743-753.

Zhang, C., X.L. Wu, Y.F. Zhao, Z.X. Deng and G.S. Qian, 2011. SIGIRR inhibits toll-like receptor 4, 5, 9mediated immune responses in human airway epithelial cells. Mol. Biol. Reports, 38: 601-609. 